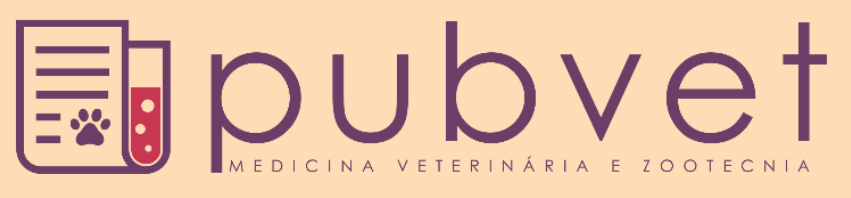

https://doi.org/10.31533/pubvet.v13n11a454.1-4

\title{
Viabilidade de isolados de Malassezia pachydermatis e Rhodotorula spp. mantidos em água destilada
}

\author{
Anna Luiza Silva ${ }^{\circ}$, Emanoele Figueiredo $\operatorname{Serra}^{2}{ }^{\theta}$, Márcia Kutscher Ripoll ${ }^{\circ}$, Stefanie \\ Bressan Waller $^{1}$, Otávia De Almeida Martins ${ }^{1 \bullet}$, Gustavo Felipe Góis Padilha Hugen ${ }^{2 * \bullet}$, \\ Renata Osório De Faria ${ }^{\bullet}$, Mário Carlos Araújo Meireles ${ }^{1}$ \\ Centro de Diagnóstico e Pesquisa em Micologia Veterinária, Setor de Doenças Infecciosas, Departamento de Veterinária Preventiva, \\ ${ }^{1}$ Faculdade de Veterinária, Universidade Federal de Pelotas. Campus Universitário, s/n, Capão do Leão, Brasil, CEP: $96010-900$. \\ ${ }^{2}$ Laboratório Regional de diagnóstico - LRD / UFPEL, RS, Brasil \\ *Autor para correspondência, E-mail: gutohugen@gmail.com
}

\begin{abstract}
Resumo. Coleções biológicas têm o objetivo de armazenar diversos organismos do ecossistema em laboratórios, para identificação e pesquisas. Para os fungos deve-se ter cautela sobre quais métodos de conservação utilizar, pois eles possuem diversos gêneros e particularidades. Dentre os métodos de estocagem, o de Castellani (água destilada) é considerado uma forma simples e com bons resultados para sua utilização no armazenamento de micro-organismos in vitro. Sendo assim, foi proposto o estudo com o intuito de avaliar a viabilidade de isolados de Malassezia pachydermatis e Rhodotorula spp. estocados pelo Método de Castellani. Foram testados quatro isolados de M. pachydermatis e três isolados de Rhodotorula spp. provenientes do Centro de Diagnóstico e Pesquisa em Veterinária (MicVet). Os isolados foram conservados em tubos de ensaio contendo água destilada estéril por seis meses em temperatura ambiente. Posteriormente os tubos foram levemente agitados e uma alíquota da solução foi semeada em placa de Petri contendo meio de cultura ideal para cada espécie fúngica. Nas condições do trabalho, todos os isolados de Rhodotorula spp. foram recuperados, enquanto nenhum isolado de $M$. pachydermatis teve crescimento. Concluindo com isso, que o método de conservação em água destilada foi viável para Rhodotorula spp., porém não é adequado para M. pachydermatis.
\end{abstract}

Palavras-chave. coleções fúngicas, leveduras, método de Castellani, micoteca

\section{Vibility of isolates of Malassezia pachydermatis and Rhodotorula spp. in distilled water}

\begin{abstract}
Biological collections has a propose to store various ecosystem organisms in laboratories for identification and research. For fungi it is necessary to be careful about which methods of conservation to use, because they have different genres and peculiarities. Among the methods of storage, Castellani (distilled water) is considered a simple form and get good results for its use in the storage of microorganisms in vitro. Therefore, it was proposed the study with the purpose of evaluating the viability of Malassezia pachydermatis and Rhodotorula spp. stored by the Castellani method. Four isolates of M. pachydermatis and three isolates of Rhodotorula spp. by the Center of Diagnosis and Research in Veterinary Medicine (MicVet). The isolates were stored in test tubes containing sterile distilled water for six months at room temperature. Subsequently the tubes were lightly stirred and an aliquot of the solution was seeded in Petri dish containing ideal culture for each fungal species. In the working conditions, all isolates of Rhodotorula spp. were recovered, whereas no isolates of $M$. pachydermatis had growth. In conclusion, the method of conservation in distilled water was viable for Rhodotorula spp., but it is not suitable for M. pachydermatis.
\end{abstract}

Keywords. fungal collections, Castellani method, micotheque, yeasts 


\section{Viabilidad de aislados de Malassezia pachydermatis y Rhodotorula spp. mantenidos en agua destilada}

Resumen. Las colecciones biológicas tienen el objetivo de almacenar diversos organismos del ecosistema en laboratorios, para identificación e investigaciones. Para los hongos se debe tener cautela sobre qué métodos de conservación utilizar, pues ellos poseen diversos géneros y particularidades. Entre los métodos de almacenamiento, el de Castellani (agua destilada) es considerado una forma simple y con buenos resultados para su utilización en el almacenamiento de microorganismos in vitro. Siendo así, se propuso el estudio con el fin de evaluar la viabilidad de aislados de Malassezia pachydermatis y Rhodotorula spp. Almacenados por el método de Castellani. Se probaron cuatro aislados de $\mathbf{M}$. pachydermatis y tres aislados de Rhodotorula spp. provenientes del Centro de Diagnóstico e Investigación en Veterinaria (MicVet). Los aislados se conservaron en tubos de ensayo que contenían agua destilada estéril por seis meses a temperatura ambiente. Posteriormente los tubos fueron levemente agitados y una alícuota de la solución fue sembrada en placa de Petri conteniendo medio de cultivo ideal para cada especie fúngica. En las condiciones del trabajo, todos los aislados de Rhodotorula spp. fueron recuperados, mientras que ningún aislado de $M$. pachydermatis tuvo crecimiento. Concluyendo con ello, que el método de conservación en agua destilada fue viable para Rhodotorula spp., Pero no es adecuado para M. pachydermatis.

Palabras clave. colecciones fúngicas, método de Castellani, levaduras, micoteca

\section{Introdução}

As coleções biológicas atuam como testemunho da heterogeneidade biológica dos ecossistemas e servem como manutenção, preservação, identificação taxonômica, catalogação, depósito e provimento para o desenvolvimento de pesquisa em ciência, em vigilância epidemiológica e biotecnologia (Silva \& Sá, 2016). Dentro das coleções biológicas existem as coleções de culturas fúngicas. Essas têm como finalidade preservar in vitro a viabilidade celular e metabólica dos fungos por longos períodos. Além disso, essas culturas também são valiosas para estudos futuros, como o desenvolvimento e produção de bioprodutos para diagnóstico, vacinas e medicamentos.

Os fungos possuem uma grande variedade, não existindo assim uma técnica padrão que seja capaz de preservá-los de forma universal e adequada. Deve-se pesquisar para cada gênero e espécie qual a melhor técnica de conservação (Santos et al., 2002).

O gênero Malassezia é constituído por leveduras lipofílicas que fazem parte da microbiota cutânea do homem e animais, podendo causar doenças de pele e otite. A única espécie não lipodependente do gênero é a $M$. pachydermatis, que faz parte principalmente da microbiota dos animais (Cruz, 2010; Guillot \& Bond, 1999). Existe uma grande dificuldade de estas leveduras serem mantidas em micotecas, devido a essas limitações, torna-se pertinente o estudo de técnicas conservativas eficazes para o gênero Malassezia (Breierová et al., 1991; Crespo et al., 2000).

Os fungos leveduriformes do gênero Rhodotorula são considerados saprófitos e contaminantes de amostras. Relatos têm citado esses como patógenos oportunistas emergentes, principalmente em pacientes imunocomprometidos. As pesquisas sobre maneiras de conservação deste fungo são importantes, pois não há muita bibliografia sobre esse assunto (Gabier et al., 2005).

Dada a complexidade do armazenamento de Malassezia pachydermatis e os poucos estudos com Rhodotorula spp., o objetivo desta pesquisa foi verificar a viabilidade dos isolados de M. pachydermatis e Rhodotorula spp. estocados segundo o método de Castellani (água destilada).

\section{Material e métodos}

No trabalho foram utilizados quatro isolados de Malassezia pachydermatis e três isolados Rhodotorula spp. provenientes do Centro de Diagnóstico e Pesquisa em Micologia Veterinária (MicVet), Faculdade de Veterinária da Universidade Federal de Pelotas. 
Tubos de ensaios contendo $3 \mathrm{ml}$ de água destilada foram auto clavados, e posteriormente em cada tubo foi colocado um dos sete isolados, o processo foi realizado em triplicata, seguindo a metodologia de Castellani. Depois de serem devidamente identificados, foram armazenados no laboratório em temperatura ambiente.

Após seis meses de estocagem, houve a verificação da viabilidade dos isolados e após uma leve agitação nos tubos e uma pequena alíquota da solução foi transferida para placas de Petri contendo meio de cultura. Os isolados M. pachydermatis foram semeados em ágar sabouraud dextrose acrescido de cloranfenicol e óleo de oliva $(\mathrm{Sb}+\mathrm{Cl}+\mathrm{Ol})$ e os isolados de Rhodotorula spp. em ágar sabouraud dextrose acrescido de cloranfenicol $(\mathrm{Sb}+\mathrm{Cl})$.

As placas foram acondicionadas em estufas a $37^{\circ} \mathrm{C}$ por dez dias, com observações diárias. As culturas foram avaliadas macro e microscopicamente, o exame direto foi realizado através de uma lâmina com o inóculo fúngico corada com fucsina e observada em objetiva de 100x e óleo de imersão.

\section{Resultados}

Após seis meses de manutenção pelo método de Castellani (Castellani, 1963), não houve recuperação dos quatro isolados de Malassezia pachydermatis. E no gênero Rhodotorula houve a recuperação dos três isolados testados no estudo, dois isolados levaram seis dias para o crescimento e um isolado cresceu em oito dias.

\section{Discussão}

O resultado obtido com a Malassezia pachydermatis corrobora com o trabalho de Crespo et al. (2000) que descreveram o método com água destilada ineficaz para esse gênero de fungo. Os autores além do estoque em água destilada testaram também o congelamento a $-80^{\circ} \mathrm{C}$, liofilização acondicionada a $-80^{\circ}$ $\mathrm{C}$ e em temperatura ambiente e repiques em diferentes meios de cultura. $\mathrm{O}$ único método bem-sucedido na manutenção de todas as espécies de Malassezia testadas, foi o congelamento a $-80^{\circ} \mathrm{C}$.

Girão et al. (2006) optaram por testar a viabilidade de cepas de M. pachydermatis armazenadas com solução salina e solução salina com óleo, pois essa solução, em comparação a água destilada, oferece melhor equilíbrio osmótico do meio. Porém esta técnica com solução salina também não se mostrou eficaz, conforme se observa com os índices de recuperação de 39,6\% e 8,4\% das cepas estocadas com seis e nove meses, respectivamente. $\mathrm{O}$ uso de óleo mineral com salina melhorou a viabilidade das cepas, em ambos os períodos de estoque aumentando para $60,4 \%$ em seis meses e $41,7 \%$ em nove meses. Segundo os autores isso ocorreu provavelmente devido à capacidade do óleo mineral em prevenir a desidratação do meio e diminuir a atividade metabólica do fungo estocado. A baixa viabilidade in vitro das Malassezia spp. constitui uma das principais dificuldades no estudo deste gênero (Crespo et al., 2000), as técnicas que apresentam resultados mais significativos são pouco acessíveis aos laboratórios de micologia, sendo eles o congelamento a $-80^{\circ} \mathrm{C}$ ou liofilização

Na literatura consultada sobre a Rhodotorula sp., poucos trabalhos discutem sobre a conservação e manutenção em micoteca desse gênero. O estudo realizado por Andreu et al. (2013) onde testaram 22 gêneros de fungos armazenados pelo método de Castellani (1939) e entre eles, três cepas de Rhodotorula spp. foram avaliadas, após 20 anos, 66,6\% (2/3) das cepas foram recuperadas.

Em seu estudo, Rodrigues et al. (1992) trabalharam com 174 gêneros de micro-organismos armazenados com o método de água destilada, dentre esses, um isolado de Rhodotorula mucilaginosa se manteve viável por 24 meses e um isolado de Rhodotorula graminis estocado por 12 meses, foi recuperado. Ficando elucidado nesse estudo que as leveduras apresentaram o melhor padrão de sobrevivência com o método de Castellani (1939) em comparação aos outros grupos avaliados.

O método de Castellani (1939) é amplamente utilizado pois apresenta fácil execução, é menos custoso e possui uma recuperação significativa dos espécimes estocados. Outras vantagens dessa técnica são que a utilizando evita-se o pleomorfismo, a contaminação com ácaros, e muitas cepas fúngicas que perdem suas características macroscópicas com repiques sucessivos, após a conservação em água destilada voltam a apresentá-las (Bueno \& Gallardo, 1998; Castellani, 1963). 
$\mathrm{Na}$ escolha do método de armazenamento deve-se levar em conta vários fatores, determinados principalmente pelo objetivo da coleção. Pois ela sendo menor e com intuito acadêmico difere de uma coleção com deposição nacional. Também se a finalidade é classificar taxonomicamente os cultivos necessitam de um método que garanta a estabilidade morfológica dos microrganismos. E uma coleção com interesse industrial terá ênfase em métodos que mantenham a estabilidade genética. Lembrando que não há uma técnica padrão, sendo cargo dos responsáveis pela micoteca escolher o melhor método para cada situação (Bueno \& Gallardo, 1998; Rodrigues et al., 1992).

\section{Conclusão}

Nas condições do presente estudo conclui-se que o método de conservação em água destilada (método de Castellani) é recomendável para armazenamento e manutenção da Rhodotorula spp. Sugerindo também, que esse não é um método indicado quando o propósito for a espécie Malassezia pachydermatis, pois, nesse trabalho não houve a recuperação dos isolados testados após seis meses.

\section{Agradecimentos}

Os autores agradecem a Coordenação de Aperfeiçoamento de Pessoal de Nível Superior (CAPES), ao Conselho Nacional de Desenvolvimento Científico e Tecnológico (CNPq), e a Fundação de Amparo à Pesquisa do Estado do Rio Grande do Sul (FAPERGS) pelo auxílio financeiro.

\section{Referências bibliográficas}

Andreu, C. M. F., Suárez, L. A. D., Zaragozí, M. T. I., López, C. A., Machín, G. M., Lancha, M. R. P. \& Gutiérrez, I. R. (2013). Conservación de cultivos de hongos de importancia médica en agua destilada. Revista Cubana de Medicina Tropical, 65(3):361-369.

Breierová, E., Kocková-Kratochvílová, A., Šajbidor, J. \& Ladzianská, K. (1991). Malassezia pachyderznatis: properties and storage: Mallassezia pachydermatis: Eigenschaften und Aufbewahrung. Mycoses, 34(7-8):349-352.

Bueno, L. \& Gallardo, R. (1998). Preservación de hongos filamentosos en agua destilada estéril. Revissta Iberoamericana de Micologia, 15(3):166-168.

Castellani, A. (1939). Viability of some pathogenic fungi in distilled water. Journal of Tropical Medicine and Hygiene, 42225-226.

Castellani, A. (1963). Further researches on the long viability and growth of many pathogenic fungi and some bacteria in sterile distilled water. Mycopathologia, 20(1-2):1-6.

Crespo, M. J., Abarca, M. L. \& Cabañes, F. J. (2000). Evaluation of different preservation and storage methods for Malassezia spp. Journal of Clinical Microbiology, 38(10):3872-3875.

Cruz, L. C. H. (2010). Micologia veterinária (Vol. 1). Rio de Janeiro: Revinter.

Gabier, A.-C., Gourdon, P., Reitz, J., Leveau, J.-Y. \& Bouix, M. (2005). Intracellular physiological events of yeast Rhodotorula glutinis during storage at+ 4 C. International Journal of Food Microbiology, 105(2):97-109.

Girão, M. D., Prado, M. R., Brilhante, R. S. N., Cordeiro, R. A., Monteiro, A. J., Sidrim, J. J. C. \& Rocha, M. F. G. (2006). Malassezia pachydermatis isolated from normal and diseased external ear canals in dogs: a comparative analysis. The Veterinary Journal, 172(3):544-548.

Guillot, J. \& Bond, R. (1999). Malassezia pachydermatis: a review. Medical Mycology, 37(5):295-306.

Rodrigues, E. G., Lírio, V. S. \& Lacaz, C. S. (1992). Preservação de fungos e actinomicetos de interesse médico em água destilada. Revista do Instituto de Medicina Tropical de São Paulo, 34(2):159-165.

Santos, I. M., Abrunhosa, L., Venâncio, A. \& Lima, N. (2002). The effect of culture preservation techniques on patulin and citrinin production by Penicillium expansum Link. Letters in Applied Microbiology, 35(4):272-275.

Silva, M. \& Sá, M. R. (2016). Coleções vivas: as coleções microbiológicas da Fundação Oswaldo Cruz. Museologia \& Interdisciplinaridade, 9(5):175-187.

Recebido: 30 de agosto, 2019.

Aprovado: 7 de novembro, 2019

Publicado: 31 de novembro,2019.
Licenciamento: Este artigo é publicado na modalidade acesso aberto sob a licença Creative Commons Atribuição 4.0 (CC-BY 4.0), a qual permite uso irrestrito, distribuição, reprodução em qualquer meio, desde que o autor e a fonte sejam devidamente creditados 